\title{
Processing of Drone's Digital Image for Determining border of Rice Fields with Edge Detection Method
}

\author{
Suhardiman $^{\mathrm{a}, *}$, Zahir Zainuddin ${ }^{\mathrm{b}}$, Salama Manjang ${ }^{\mathrm{c}}$ \\ aDepartment of Electrical Engineering, Engineering Faculty, Hasanuddin University. Email:diman@unhas.ac.id \\ bepartment of Informatics, Engineering Faculty, Hasanuddin University. Email:zahir@unhas.ac.id \\ cDepartment of Electrical Engineering, Engineering Faculty, Hasanuddin University. Email:salamamanjang@unhas.ac.id
}

\begin{abstract}
Edge detection was the basic thing used in most image processing applications to get information from the image frame as a beginning for extracting the features of the segmentation object that will be detected. Nowadays, many edge detection methods create doubts in choosing the right edge detection method and according to image conditions. Based on the problems, a study was conducted to compare the performance of edge detection using methods of Canny, Sobel and Laplacian by using object of rice field. The program was created by using the Python programming language on OpenCV. The result of the study on one image test that the Canny method produces thin and smooth edges and did not omit the important information on the image while it has required a lot of computing time. Classification is generally started from the data acquisition process; pre-processing and post-processing. Canny edge detection can detect actual edges with minimum error rates and produce optimal image edges. The threshold value obtained from the Canny method was the best and optimal threshold value for each method. The result of a test by comparing the three methods showed that the Canny edge detection method gives better results in determining the rice field boundary, which was $90 \%$ compared to Sobel $87 \%$ and Laplacian $89 \%$.
\end{abstract}

Keywords: Canny; edge detection; drone; rice field

\section{Introduction}

The agricultural sector has a very important role in national development because it utilizes enormous natural and human resources. Rice field is agricultural land that is patched and bounded by a dike, a channel to hold or distribute water, which is usually planted with rice [1]. Agricultural land in the form of rice fields is usually characterized by the presence of embankments surrounding it to restrict between the rice field and the other one. Besides, the embankment of the rice field is also made to prevent an excessive influx of water so that the water conditions can be adjusted as needed. Rice fields have many functions, both for human life and the environment [2]. As technology develops, so many developments and innovations were carried out for research, especially in the agricultural sector in the rice fields like the detection of changes in paddy planting patterns [3], and detection of flood zoning in paddy fields using satellite imagery $[4,5]$.

${ }^{*}$ Corresponding author. Tel.: +62-852-9961-1858

Jalan Poros Malino km. 6 Bontomarannu

Gowa, Sulawesi Selatan, Indonesia, 92171
Availability of advanced computer vision technology with the increase in the computing capabilities of the computer that facilitate the development of automated farming systems be able to solve problems, effectively increase flexibility and efficiently made a lot of research done [6].

The study conducted by Kania, et al. [7] who monitor rice phenology and planting schedules in very large paddy fields by using satellite images and the method used with the results of the system that can detect changes in the phenology stage of paddy with a composite time series of 8 days, planting and harvest date while the system still has to be developed to produce a good correlation in the detection system. The research conducted by $\mathrm{Yi}$-Hsing, et al. a remote sensing technology for automatic detection of rice fields using the Profile Matching (PM) method, peak Detection (PD), and difference classification (DC) on multi-temporal images with results of accuracy $20 \%$ good results Enhancement when using large images [8].

Edge detection was a basic thing that used in most image processing applications to get information from image frames as a beginning for features extraction of segmentation objects that will be detected. The edge detection segmentation process can be used to detect object 
lines and boundaries between objects by detecting discontinuities in one image [9]. Research conducted by Lakshmi, et al. [10] use Canny edge detection to find the closest edge of the object to maximize the location of the pixel size in the image. While research by Ambarwati et al. [11] using Canny edge detection to detect the image side of a former mine, in this study, results show that Canny is an edge detection algorithm that provides good edge detection results that can localize the right edge points.

The development of an area will be accompanied by changes in geographic information. It requires a long process to be able to recreate geographic information. By observation, inventorying quality and environmental conditions, if carried out by field surveys, sometimes can not follow the significant growth of geographic information changes. The drone's image data can use to follow the growth of geographic information changes.

Problem-related to the agricultural sector is the lack of information about these rice fields. This information can include the boundaries of rice fields, ownership of rice fields, plants, as well as the areas affected by floods and pests. The determination of plots of rice fields usually relies on direct observation to see one by one from each plot of rice fields. This manual process takes a long time and is not efficient. To increase the productivity in the agricultural sector, drones are used to extract data and automation technology in the process of detecting rice fields to find the boundaries of each plot out with the resulting picture. This study used the pre-processing image method and image segmentation technique in image processing that using transformation BGR to GRAY color. The result of color transformation would be used in the segmentation phase for determining the boundary of the rice field.

\section{Literature Review}

\subsection{Canny Operator}

Canny is one of the modern edge detection algorithms. In 1986, John Canny proposed three criteria for optimizing edge detection in images. These criteria are [12]:

- Good detection, the purpose of this criterion is to maximize the signal to noise ratio (SNR) so that all edges can be detected properly or nothing is undetected.

- Good localization. The edge detected by the operator must be as close as possible to the center of the actual edge.

- One response to a single edge. The detector does not provide an edge that is not the actual edge.

The optimal edge detection algorithm can be obtained by combining the Canny criteria above. Primarily, the Canny algorithm consists of six parts [12]:

- Processing original images into gray images. Using images in the GBR format, for example, the gray conversion formula can be seen as follows:

$$
g(x, y)=0.299 R+0.587 G+0.114 B
$$

- With a Gaussian smoothing template, the original data is convoluted. The produced image is slightly blurred from the original one.

- $G_{x}$ and $G_{y}$ can be calculated using a template. The gradient magnitude is calculated using Eq. (2) and the direction angle gradient is calculated using Eq. (3).

$$
\begin{gathered}
\operatorname{mag}(\nabla f)=\left[G_{x}^{2}+G_{y}^{2}\right]^{1 / 2} \\
\phi(x, y)=\arctan \left(\frac{G_{y}}{G_{x}}\right)
\end{gathered}
$$

- The pixel corresponding to the local gradient of nonmaximum is determined. This pixel is suspended by a higher threshold, and it is considered as the wrong edge.

- With the lower threshold, the boundary initial pixels are searched, and new edge pixels are collected according to the pixels sought. Finally, the entire edge of the image is closed.

- The edges of the image are tracked based on the edges of the pixels found.

\subsection{Sobel Operator}

The operator of Sobel is based on the first-order differential. This operator combines images with small integer filters that can be separated in horizontal and vertical directions. Computationally, it is not expensive. This is a type of orthogonal gradient operator which is a partial derivative in the $\mathrm{x}$ and $\mathrm{y}$ direction calculated using the environment $3 \times 3$ from $f(x, y)$, as follows [13]:

$$
\begin{gathered}
S x=\{f(x+1, y-1)+2 f(x+1, y)+f(x+1, y+1)\} \\
-\{f(x-1, y-1)+2 f(x-1, y)+f(x-1, y+1)\} \\
S x=\{f(x-1, y+1)+2 f(x, y+1)+f(x+1, y+1)\} \\
-\{f(x-1, y-1)+2 f(x, y-1)+f(x+1, y-1)\}
\end{gathered}
$$

The gradient in the oblique direction:

$$
g(x, y)=\sqrt{S_{x}^{2}+S_{y}^{2}}
$$

For digital images, the formula above can be reduced to the following:

$$
g(x, y)=|S x|+|S y|
$$

Convolution operator:

$$
\left[\begin{array}{ccc}
-1 & 0 & -1 \\
-2 & 0 & 2 \\
-1 & 0 & 1
\end{array}\right]
$$




$$
\left[\begin{array}{ccc}
-1 & -2 & -1 \\
0 & 0 & 0 \\
1 & 2 & 1
\end{array}\right]
$$

There are two main advantages of Sobel edge detection: (a) It has a soothing effect on unwanted noise in an image, (b) Because 2 lines or 2 columns differential has been used, it increases the edge element until the edges become thick and bright.

\subsection{Laplacian Operator}

Laplacian is a scalar differential operator for scalar functions $\phi$ defined by [14]

$$
\Delta f=\nabla^{2} f=\nabla \cdot \nabla f
$$

Laplacian is used in digital image processing for edge detection purposes because it shows an area of rapid intensity change. There are two types of Laplacian operators:

- Positive Laplacian Operator: This operator consists of a standard mask where the angle element must be zero and the middle element must be negative from the mask.

$$
\left(\begin{array}{lll}
0 & 1 & 0 \\
1 & 4 & 1 \\
0 & 1 & 0
\end{array}\right)
$$

- Negative Laplacian Operators. This operator consists of a basic mask where the angle element must be zero and the middle element must be positive from the mask and the remaining element must be -1 . The edges in the image are taken in a positive Laplacian operator.

$$
\left(\begin{array}{ccc}
0 & -1 & 0 \\
-1 & 4 & -1 \\
0 & -1 & 0
\end{array}\right)
$$

\subsection{Transformation of RGB Colour to Grayscale}

The use of grayscale images is because it requires a little information given to each pixel compared to the color image. The gray color in the grayscale image is R (Red), G (Green), $\mathrm{B}$ (Blue) color which has the same intensity. In grayscale image only requires a single intensity value compared to the color image requires three intensities for each pixel. The intensity of grayscale images is stored in 8-bit integers which gives 256 possibilities which start from level 0 to 255 ( 0 for black and 255 for white and the value between them is the degree of gray). RGB color transformation to Grayscale can be done by taking the average values of $r$, $g$, and $b$ so that with the following equation [15].

$$
S=\frac{r+g+b}{3}
$$

\subsection{Threshold}

Thresholding is the process of converting grayscale images into a binary image, which means images in black and white color. The equation used for the threshold process.

$$
g(x, y)=\left\{\begin{array}{l}
0, f(x, y)<1, \\
1, f(x, y) \geq 1
\end{array}\right.
$$

Where $g$ is the result of the Thresholding binary image and $(x, y)$ is the position of each pixel. If the image pixel $f$ $(\mathrm{x}, \mathrm{y})$ is above the threshold value, the pixel will be changed to white and the value will be 1 . If the image pixel $f(x, y)$ has the same value or is a level below the threshold value, so the pixel is changed to black and the value becomes 0 [16].

\section{Proposed Method}

\subsection{Data Input}

The input data used in this study is in images form. The picture was taken using a camera of DJI Phantom 3 Pro drone with resolution 12 Megapixel with a height of 50 meters from the ground with a $90^{\circ}$ camera tilt angle facing down. Image data of rice fields are taken directly in Bone Regency, South Sulawesi, Indonesia. Fifty pictures were taken and then mosaicking to combine them to produce 1 image with resolution 14235 x 12725 pixels. The scenario for taking image data can be seen in Fig. 1.

\subsection{General System Design}

The proposed system is a system for detecting rice fields, images were taken by a drone device, then the Image processing process will be carried out; pre-processing and image segmentation techniques. Figure 2 shows the rice field plot system.

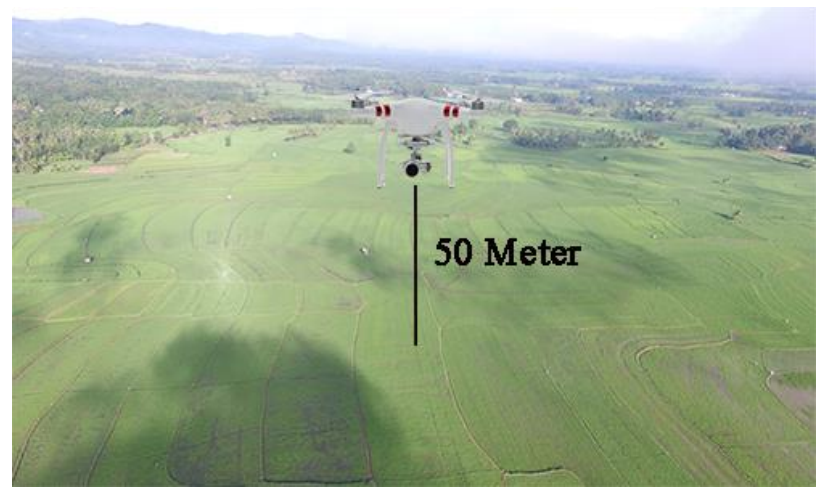

Figure 1. Shooting scenario

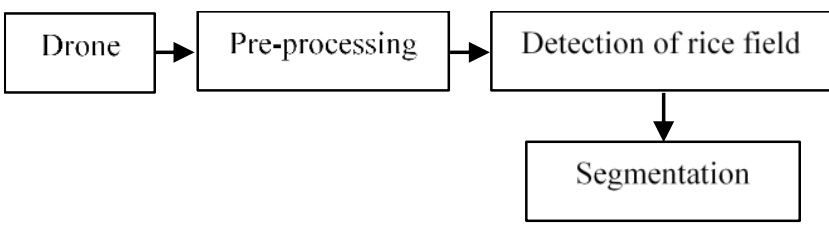

Figure 2. General system design. 


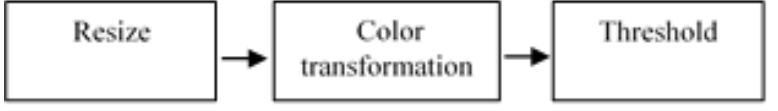

Figure 3. Preprocessing Design

\subsection{Preprocessing Design}

To be more effective, the process carried out is using image processing technique as a solution to minimize errors in visual detection of human eyes. However, the research focus to be carried out in this study is at the preprocessing stage for the detection of rice fields; the BGR color transformation process which is transformed into a Grayscale image for further processing with binary image threshold. The preprocessing design can be seen in Fig. 3 .

The process of image acquisition is the stage of taking objects at the initial step of image processing. In this study, the image of rice fields will be taken using a drone's camera.

- Resize: Image from the camera of a drone device have a large size, so the system takes a long time to process it. Image size will be reduced without affecting its quality.

- Color Transformation: The resized image is transformed from RGB color to Grayscale.

- Threshold: After color transformation to grayscale, then the threshold process is carried out. In the threshold process, the pixel value that meets the threshold is mapped to a satisfying value. The threshold value used in this study is 125 . So, all pixels in a range of less than 125 will be worth 0 , while for all pixels in the range 125 255 are worth 1.

\subsection{Segmentation Design}

- Canny

The first process is the thinning of the image to eliminate the noise with the gaussian filter. The results of the thinning process are used for the process of obtaining edge strength with the gaussian operator like in Eq. (2). For edge strength results, previously used for the process of removing dotted lines in the image. Therefore, to help edge tracking, the Gx and Gy gradients are each calculated by the matrix operator CannyMask $3 \times 3$.

\section{- Sobel}

The process of Sobel detection, which given one zero buffer. The advantage of this Sobel method is the ability to reduce noise before making detachment calculations. The kernel filters used in the Sobel method are shown in Table 2.

Table 1. Matrix 3x3 Canny Operator

\begin{tabular}{|c|c|c|c|c|c|c|c|}
\hline \multirow{3}{*}{$\mathrm{G}_{\mathrm{x}}$} & 1 & 0 & -1 & \multirow{3}{*}{$\mathrm{G}_{\mathrm{y}}$} & -1 & -1 & -1 \\
\hline & 1 & 0 & -1 & & 0 & 0 & 0 \\
\hline & 1 & 0 & -1 & & 1 & 1 & 1 \\
\hline
\end{tabular}

Table 2. Matriks 3x3 Sobel Operator

\begin{tabular}{|c|c|c|c|c|c|c|c|c|}
\hline \multirow{3}{*}{$\mathrm{S}_{\mathrm{x}}=$} & -1 & 0 & 1 & \multirow{3}{*}{ and } & \multirow{3}{*}{$S_{y}=$} & 1 & 2 & 1 \\
\hline & -2 & 0 & 2 & & & 0 & 0 & 0 \\
\hline & -1 & 0 & 1 & & & -1 & -2 & -1 \\
\hline
\end{tabular}

- Laplacian

The Laplace operator detects edge location more accurately, especially on steep edges. The beginning process is blur image using a gaussian, then find zerocrossing of Laplacian and compare it with the threshold.

\section{Results and Discussion}

The process of determining rice field boundaries with edge detection starting in preprocessing. The images taken by a drone device camera have a large size, the system needs a long time to process it. Therefore, the resize process is carried out to change the size of the image without affecting its quality. The results of the image resize process are shown in Fig. 4(a).

Next, the resized picture is converted to a grayscale image by transformation color. The result of the color transformation process to the grayscale image can be seen in Fig. 4(b). The results of the color transformation process, then become the input to the image threshold process with a threshold value of 125 as a threshold value to obtain a binary image. The result can be seen in Fig. 4(c).

After the preprocessing process, the edge detection process is then carried out using Canny, Sobel, and Laplacian operators. The results are shown in Figs. 5(a) to (c).

- Canny

Based on the Canny edge detection process, this study uses gradient $\mathrm{G}(\mathrm{x}, \mathrm{y})$ which is a vector consisting of two elements, namely Gx and Gy. Edge detection in this process is done by reading each pixel on the image by reading the pixel from the top-left pixel and moving to the bottom right pixel. The results of the edge detection process using Canny edge detection can detect $90 \%$ rice field boundary as shown in Fig. 5(a).

- Sobel

In this process to detect edge is to read each pixel on the image by reading from the leftmost pixel and moving to the bottom right pixel. Therefore, to guide edge tracking, the Gx and Gy gradients are each calculated by the matrix of Sobel Mask3x3 method. The result of Edge detection using the Sobel operator can detect rice field plot boundaries by $87 \%$ as shown in Fig. 5(b).

- Laplacian

The Laplacian operator in this study is looking for zero values in the second derivative of the image because when the first derivative is at the maximum value, the second derivative will produce a zero value. The second derivative of the $\mathrm{x}$ and $\mathrm{y}$ direction are combined into one operator value. This value is represented in the form of a sum of two convolution matrices from the $\mathrm{x}$ and $\mathrm{y}$ directions. If the threshold meets, then it is expressed as an edge. The results of the Laplacian operator process can detect the rice plot boundary by $89 \%$ can be seen in Fig. 5(c). 


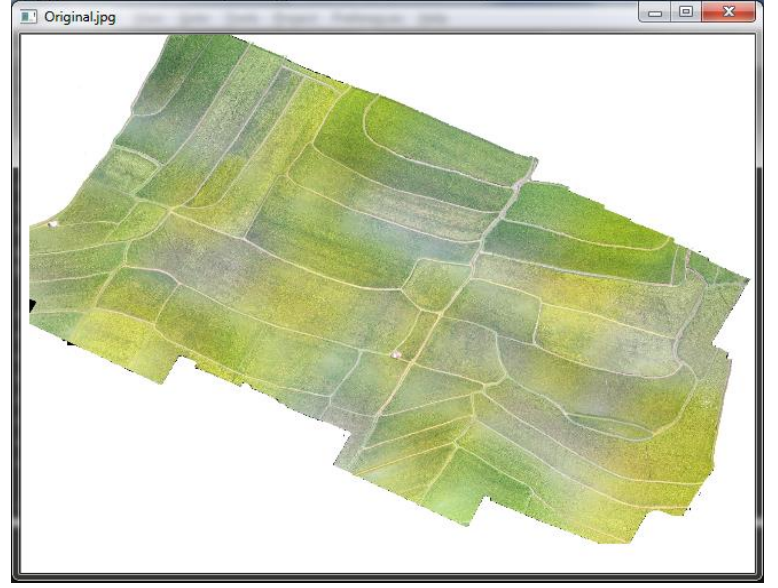

(a) Resized Picturer

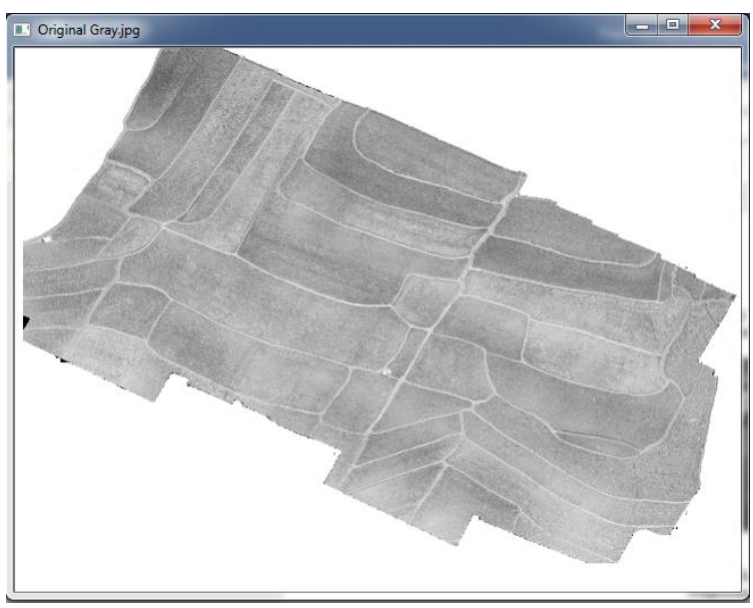

(b) The image has been made a color transformation

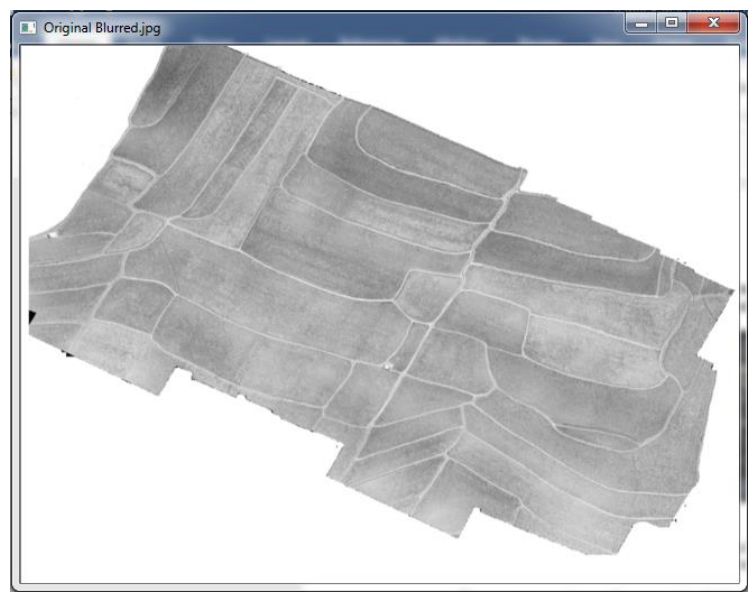

(c) Picture by threshold

Figure 4. Preprocessing process for determining rice field plot boundaries

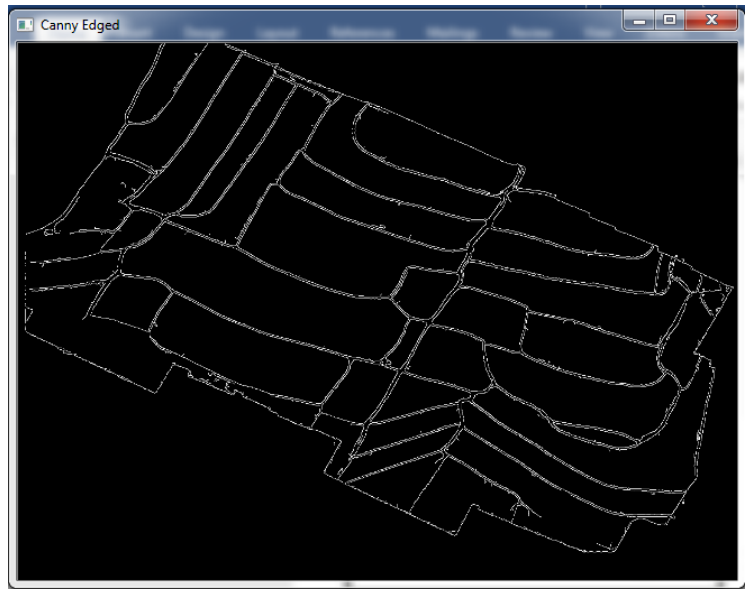

(a) The result of Canny edge detection

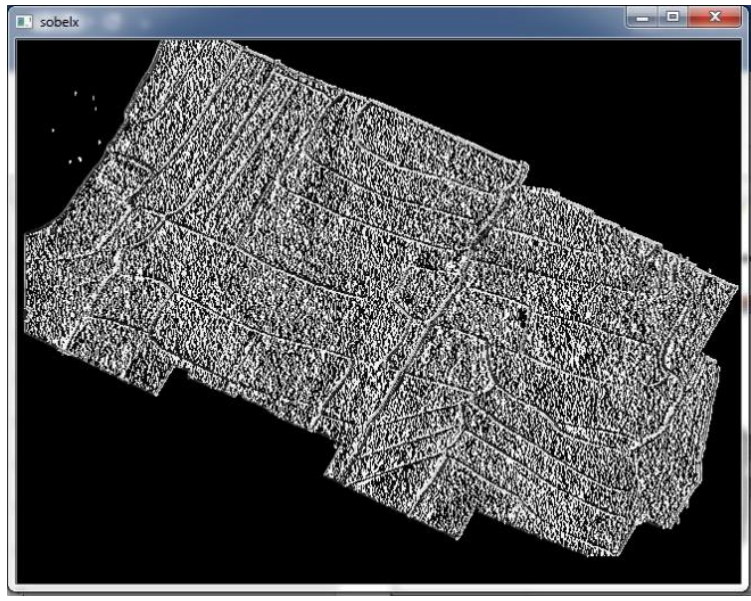

(b) Result of Sobel edge detection

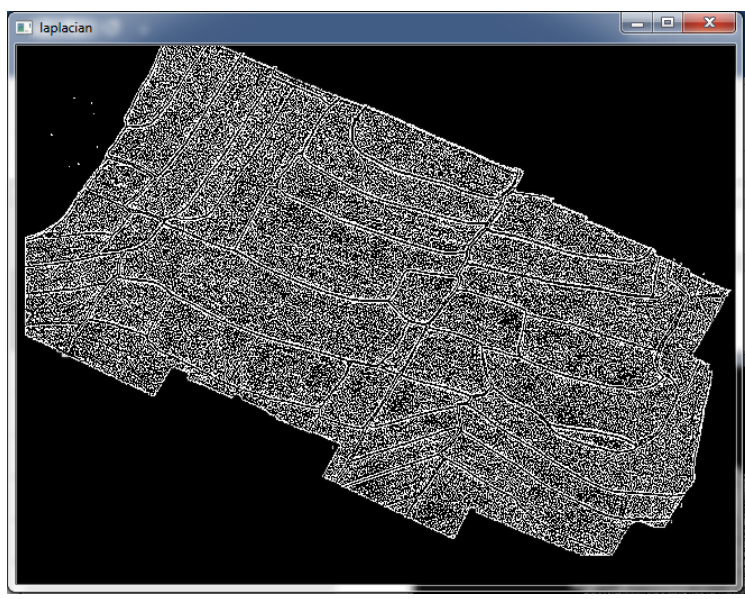

(c) The result of Laplacian edge detection

Figure 5. The result Laplacian edge detection 


\section{Conclusion}

This research used data of the image taken in Kahu District, Bone Regency, South Sulawesi. Image captured by a DJI Phantom 3 Pro camera drone with 12 Megapixel resolution with a height of 50 meters from the ground with $90^{\circ}$ camera tilt angle facing down. The edge detection method used to determine the boundary of rice fields, namely Canny, Sobel, and Laplacian. The test results by comparing the three methods showed that the Canny edge detection method gives better results in determining the rice field boundary, which $90 \%$ compared to Sobel $87 \%$ and Laplacian 89\%.

\section{References}

[1] Badan Pusat Statistik. [Online]. Available: https://www.bps.go.id/istilah/index.html?Istilah_sort=deskripsi_ind\&I stilah_page=23. [Accessed: 04-Jul-2019].

[2] Sudrajat, Knowing Rice Fields and Understanding Its Functions for Humans and the Environment. Gadjah Mada University Press, 2015. [in Bahasa]

[3] D. R. Pratama, Detection of Changes in Paddy Planting Patterns in East Java Based on MODIS Imagery Using the Cross Correlation Method, 2017. [in Bahasa]

[4] L. Rahmadany, A. L. Nugraha, B. Sasmito, and N. Febrianti, Jurnal Geodesi Undip Oktober, 2014, vol. 3, p. 7, 2014.

[5] Flood risk zonation Pre-monsoon land use and land cover Land, Research Gate. [Online]. Available: https://www.researchgate.net/figure/Flood-risk-zonation-Premonsoon-land-use-and-land-cover-Land-use-and-land-cover-mapof_fig4_294089869. [Accessed: 09-Jul-2019].

[6] X. Li and W. Zhu, "Apple Grading Method Based on Features Fusion of Size, Shape, and Color," Procedia Eng., vol. 15, pp. 2885-2891, 2011.
[7] D. K. Sari, I. H. Ismullah, W. N. Sulasdi, and A. B. Harto, "Detecting Rice Phenology in Paddy Fields with Complex Cropping Pattern Using Time-Series MODIS Data," J. Math. Fundam. Sci., vol. 42, no. 2, pp. 91-106-106, 2010.

[8] G. World, "Automatic detecting rice fields by using multitemporal satellite images, land-parcel data and domain knowledge," Geospatial World, 11-Sep-2009.

[9] G. M. H. Amer and A. M. Abushaala, "Edge detection methods," in 2015 2nd World Symposium on Web Applications and Networking (WSWAN), Sousse, Tunisia, 2015, pp. 1-7.

[10] S. Lakshmi and Dr. V. Sankaranarayanan, "A study of Edge Detection Techniques for Segmentation Computing Approaches," Int. J. Comput. Appl., vol. CASCT, no. 1, pp. 35-41, Aug. 2010.

[11]A. Ambarwati and R. Passarela, "Segmentasi Citra Lahan Bekas Tambang Terbuka Menggunakan Metode Deteksi Tepi Canny,”p. 9, 2017.

[12] X. Yan and Y. Li, “A Method of Lane Edge Detection Based on Canny Algorithm," p. 5.

[13]A. Kalra and R. L. Chhokar, "A Hybrid Approach Using Sobel and Canny Operator for Digital Image Edge Detection," in 2016 International Conference on Micro-Electronics and Telecommunication Engineering (ICMETE), Ghaziabad, India, 2016, pp. 305-310.

[14] R. Bansal, G. Raj, and T. Choudhury, "Blur image detection using Laplacian operator and Open-CV," in 2016 International Conference System Modeling \& Advancement in Research Trends (SMART), Moradabad, India, 2016, pp. 63-67.

[15]Y. Wan and Q. Xie, "A Novel Framework for Optimal RGB to Grayscale Image Conversion," in 2016 8th International Conference on Intelligent Human-Machine Systems and Cybernetics (IHMSC), Hangzhou, China, 2016, pp. 345-348.

[16]M. F. N. Wahidah, N. Mustafa, M. Y. Mashor, and S. S. M. Noor, "Comparison of color thresholding and global thresholding for ZiehlNeelsen TB bacilli slide images in sputum samples," in 2015 2nd International Conference on Biomedical Engineering (ICoBE), Penang, Malaysia, 2015, pp. 1-6. 